\title{
Linx
}

LinX Revue des linguistes de l'université Paris X Nanterre

$62-63 \mid 2010$

Entre syntaxe et discours. Éclairages

épistémologiques et descriptions linguistiques

\section{La construction en avoir SN qui SV (« j’ai ma copine qui habite à Paris ») : une forme de dispositif clivé ?}

\section{Virginie Conti}

\section{(2) OpenEdition}

\section{Journals}

Édition électronique

URL : http://journals.openedition.org/linx/1353

DOI : $10.4000 /$ linx.1353

ISSN : 2118-9692

\section{Éditeur}

Presses universitaires de Paris Nanterre

Édition imprimée

Date de publication : 1 juillet 2010

Pagination : 63-87

ISSN : 0246-8743

\section{Référence électronique}

Virginie Conti, «La construction en avoir SN qui SV («j'ai ma copine qui habite à Paris ») : une forme de dispositif clivé ? », Linx [En ligne], 62-63 | 2010, mis en ligne le 04 décembre 2013, consulté le 20 avril 2019. URL : http://journals.openedition.org/linx/1353 ; DOI : 10.4000/linx.1353 


\title{
La construction en avoir SN qui SV ("j'ai ma copine qui habite à Paris ») : une forme de dispositif clivé ?
}

\author{
Virginie Conti \\ Université de Neuchâtel \\ virginie.conti@unine.ch
}

\section{Introduction}

L'étude de la construction en [il y a SN qui SV], parfois décrite comme un "gallicisme $»^{2}$, est un peu un classique des analyses du français contemporain. En observant les corpus de français parlé, on se rend compte que le verbe avoir fournit un autre outil souvent utilisé, mais moins souvent décrit. Le verbe avoir y figure sous une forme personnelle cette fois, et la construction est considérée par certains linguistes comme une sorte de variante de celle en il y $a$; les exemples ${ }^{3}$ (1) à (5) illustrent la construction qui sera l'objet de cette étude.

\footnotetext{
${ }^{1}$ L'intervention orale à la source de cet article a eu lieu dans le cadre de mon engagement sur le projet FNS n¹00012-126745 «La structure interne des périodes - nouveaux développements » sous la direction de Marie-José Béguelin.

${ }^{2}$ Cf. par exemple Léard (1992). L'appellation «gallicisme» n'est pas une catégorie linguistique utilisable, mais montre que cette structure en il y a est bien identifiée comme une structure fréquente en français.

${ }^{3}$ Les exemples proposés ici ont deux sources. Beaucoup sont tirés du corpus CRFP (DELIC, 2004) : j'y ai cherché toutes les formes du verbe avoir (= à toutes les personnes) à l'indicatif présent,
} 
Virginie Conti

(1) j'ai ma copine qui habite à Paris (CRFP, PRI-BRI-1)

(2) j'ai ma collègue qui arrive à deux heures (CRFP, PRI-CLE-3)

(3) j'ai mon pain au chocolat qui me fait mal à l'estomac (oral)

(4) tu as ton huile qui est ouverte (oral)

(5) il a son cours qui recommence à deux heures il a pas envie d'arriver en retard (oral)

On rencontre des illustrations de cette construction à toutes les personnes, avec probablement une fréquence plus haute à la $1^{\text {re }}$ personne du singulier, mais aussi beaucoup d'exemples en $t u$ ou en vous dans certains genres de discours.

On voit que la construction répond à un «schéma général » de forme

[pronom - verbe avoir - SN - qui - SV].

Et elle a en plus la particularité suivante: la séquence en [qui - SV], généralement appelée "relative », n'est pas une relative adjective mais une séquence prédicative. Dans les exemples (1) à (5) ci-dessus, le segment à forme de relative constitue un prédicat dans l'énoncé : son contenu n'est pas présupposé mais bien posé sur un mode attributif ${ }^{4}$ - c'est là une caractéristique essentielle de cette structure. Le même «schéma général » peut en effet aussi être représenté par des exemples avec $\left[J^{\prime} A I^{5}-\mathrm{SN}\right]$ suivi d'une 'simple' relative adjective, comme (6). Ce type d'exemple relève à l'évidence d'une autre analyse.

(6) eux ils ont le logiciel qu'on a développé (CRFP, PRO_PSE, exemple simplifié)

Ce «schéma général » recouvre ainsi diverses constructions, ayant chacune une analyse syntaxico-sémantique différente. Comme évoqué plus haut, celle qui m’intéressera ici est celle qui est illustrée par les exemples (1) à (5) et répond aux critères mentionnés ci-dessus (appartenance à ce schéma général + séquence à forme de relative ayant une fonction prédicative). Elle est souvent décrite comme présentant une relative prédicative, et

imparfait, futur, et j’ai relevé celles qui étaient suivies d'une «relative », et correspondaient à la structure qui m’intéresse. J'ai par ailleurs quelques dizaines d'exemples de la structure relevés dans divers écrits au hasard de mes lectures, ou entendus dans des conversations, conférences, médias oraux, etc. Pour ceux-ci, j'ai simplement indiqué entre parenthèses « oral », « internet », ou « courriel » (le contexte d'apparition des exemples oraux n'est bien sûr pas accessible, puisque ces exemples ont été transcrits 'au vol' par mes soins, sur le moment et sans enregistrement). Tous les exemples cités sont authentiques ; toute manipulation est explicitement marquée.

${ }^{4}$ Cf. Lagae \& Rouget, 1998 : elles proposent des tests permettant de montrer qu'une relative n'est pas adjective mais prédicative.

${ }^{5}$ J'utiliserai ici la forme AVOIR pour évoquer aussi bien les formes personnelles du verbe que les formes en il y a. La forme J'AI renvoie quant à elle uniquement aux formes personnelles, et donc exclut il y a (les petites majuscules indiquent que la forme de $1^{\text {re }}$ personne ne fait que représenter l'ensemble possible de toutes les personnes). 
est souvent rapprochée des exemples en [il y a SN qui SV] ; on devra donc se situer par rapport à ces deux aspects.

Cet article vise à proposer une description de la construction en question, et plus particulièrement une description syntaxique - on verra toutefois que cette dernière ne pourra être totalement détachée des aspects liés à l'analyse discursive, et se trouve donc en fait à l'interface entre la syntaxe et le discours.

La présente contribution s'articule en trois points. (1) Je synthétiserai tout d'abord les principales descriptions de la construction existant dans la littérature scientifique, en me centrant sur les aspects syntaxiques ${ }^{6}$. Je relèverai alors les points qui me paraissent problématiques dans ces descriptions, et les discuterai ; je proposerai des pistes d'analyse alternatives. (2) Je problématiserai de façon plus approfondie les pistes évoquées, en discutant les notions de construction clivée et de dispositif syntaxique, pour voir si elles peuvent s'appliquer à mon objet d'étude. (3) Enfin, je montrerai, à partir d'exemples contextualisés, en quoi l'analyse de la structure étudiée requiert un traitement parallèle des dimensions syntaxique et discursive. Certains paramètres de la construction seront alors étudiés de plus près, et l'on verra plus précisément quels types d'exemples répondent à la description syntaxique proposée, et quels types n'y répondent pas.

\section{Description des séquences $\left[J^{\prime} A I-\mathrm{SN}-q u i-\mathrm{SV}\right]$}

Si on la compare à d'autres constructions fréquentes en français oral, cette structure a été encore relativement peu décrite dans la littérature linguistique. Je propose ici un petit tour d'horizon ainsi qu'une discussion générale des descriptions existantes.

\subsection{Descriptions existantes}

On peut classer la plupart des descriptions disponibles dans une rubrique de type « explication par la prédication seconde». Elles correspondent en effet à une analyse qui fait du verbe avoir un verbe constructeur, et qui considère qu'il y a une double prédication dans ces énoncés (en schématisant : la première prédication est constituée d'avoir + le SN «objet direct»; la seconde est formée par le SV de la «relative prédicative »). Voyons (1.1.1 à 1.1.4) un peu plus spécifiquement les descriptions évoquées ; je les discuterai au point 1.2, et présenterai alors les pistes proposées par les quelques descriptions alternatives que l'on peut trouver dans la littérature.

1.1.1 Rothenberg (1979) est probablement la première à s'être intéressée de près aux constructions en [J'AI - SN - qui - SV]. Elle en propose quelques exemples attestés (cf. ici (7)-(9), < ibid., p. 356), et signale la grande proximité de cette construction avec une construction parallèle, dans laquelle le référent du SN est dans une relation de partie à tout avec le référent du sujet du verbe avoir (cf. (10), < ibid.).

\footnotetext{
${ }^{6}$ Dans l'espace restreint de cet article, l'accent sera mis sur l'analyse proprement syntaxique de la construction étudiée ; une description plus globale, montrant plus précisément, à partir de nombreux exemples, les diverses contraintes qui la caractérisent, est proposée dans ma thèse (Conti, en cours).
} 
(7) J'ai mon fils Etienne et ma fille qui connaissent bien l'écriture (< René Bazin, dans Sandfeld ${ }^{7}$ )

(8) ...non pas aujourd'hui. J'ai Jenny qui m'attend au d'Harcourt (< Audiberti, dans Sandfeld)

(9) Nous avons beaucoup de nos clients qui nous en ont parlé (< Lavedan, dans Sandfeld)

(10) Il a les cheveux qui tombent.

Elle met ensuite en évidence des contraintes qui pèsent sur la construction en question (ibid., p. 357) :

— «Le sujet du verbe AVOIR désigne toujours un être ANIMÉ. »

— L’antécédent doit effectuer une référence au sujet. «J'ai ma femme (ma fille, mon amie) qui m'attend, mais non pas *J'ai votre cousine qui m'attend. $\gg$

— Le pronom relatif doit être qui.

— Le verbe de la relative peut dénoter une activité ou un état.

— Il peut y avoir une négation dans la relative ; il ne peut en revanche y en avoir une qui porte sur le verbe avoir.

— Les temps verbaux possibles sont les suivants : présent ou imparfait dans les deux propositions (situation la plus fréquente); éventuellement passé composé dans la relative. ${ }^{?}$

Du point de vue syntaxique, l'auteur décrit ces énoncés comme des constructions à relative prédicative : la relative, obligatoire $i^{1} i^{10}$, est pour elle « un cas particulier de second élément d'un syntagme prédicatif» (ibid., p. 354), qui en l'occurrence se trouve «en position postverbale directe après avoir» (ibid., p. 356). Ainsi, la construction s'analyse comme un verbe avoir ${ }^{11}$ suivi directement d'un syntagme prédicatif contenant lui-même une relative prédicative, obligatoire.

\footnotetext{
${ }^{7}$ Sandfeld lui-même considère cette construction comme présentant une «proposition relative attribut » $(1936: 143)$.

${ }^{8}$ La référence n'est pas toujours explicite. «Dans J'ai Jenny qui m'attend il y a référence implicite à une relation avec le sujet (ma femme, ma fille, quelqu'un à qui j'ai donné rendez-vous) » (Rothenberg 1979, p. 357).

${ }^{9}$ Plusieurs de ces contraintes sont relativisées par certains de mes exemples de corpus, et demanderaient donc encore discussion (cf. Conti, en cours).

10 Cette caractéristique l'oppose aux relatives qu'elle nomme attributives (qui, elles, sont facultatives), qu'elle examine dans la seconde partie de son article (Il est là qui pleure; Marie le voit qui attend, cf. Rothenberg 1979, p. 362-391).

${ }^{11}$ Le statut exact de ce verbe dans la construction, et la nature de la relation qu'il entretient avec le $\mathrm{SN}$ ou avec tout le syntagme prédicatif, n'est malheureusement pas très explicite chez Rothenberg (cf. discussion plus bas, point 1.2).
} 
1.1.2 Furukawa, entre ses différents écrits (1987, 1996, 2002, 2005), semble avoir un peu changé d'avis sur l'analyse qu'il faut donner selon lui aux structures en [J'AI-SN - qui - SV]. Dans sa publication de 1996, des exemples comme (11) étaient décrits comme illustrant une structure bipropositionnelle bithématique schématisée ainsi : $\left[\mathrm{p} 1\right.$ avoir $\left(\mathrm{p}_{2} \mathrm{SN}\right]$ qui $\left.\mathrm{V}\right)$.

(11) J'ai mon petit qui est malade (Furukawa, 1996, p. 88)

Le pronom/SN régissant avoir constitue alors le premier thème ou le «cadre thématique de la phrase» $(1996$, p. 94), et le SN qui suit avoir un second thème, plus précis, dont il sera prédiqué quelque chose. Le rôle de la construction en avoir est d'être « un appareil présentateur de double thème » (ibid., p. 95); $\mathrm{P}_{1}$ et $\mathrm{P}_{2}$ ensemble forment en effet pour lui « une construction bithématique » (ibid.). Au niveau sémantique, c'est la seconde proposition qui forme la prédication principale, alors qu'elle formait la prédication subordonnée au niveau syntaxique.

Dans son ouvrage de 2005, il redéfinit les choses et, en discutant d'exemples pourtant semblables, n'accorde plus à la structure en question le statut de véritable construction grammaticale bithématique : il la juge en fait sous-déterminée à cet égard.

(12) Elle a son mari qui est malade. (Furukawa, 2005, p. 32)

(13) J'ai mon ami qui m'attend. (ibid.)

Certains énoncés en [J'AI - SN - qui - SV] peuvent encore être bithématiques (c'est le cas de (12)- (13)), mais en quelque sorte par accident, et non plus par définition ${ }^{12}$.

La description proprement syntaxique des structures étudiées n'est pas très explicite, les intérêts de Furukawa semblant être plutôt de nature sémantique. On comprend toutefois qu'il continue de placer cette analyse dans le cadre de la prédication seconde, et qu'il rejoint donc sur ce point les trois autres auteurs de cette section.

1.1.3 Lagae \& Rouget, dans leur article de 1998 consacré aux relatives prédicatives, relèvent elles aussi, et parfois en suivant Rothenberg, certaines contraintes s'appliquant aux structures en $\left[J^{\prime} A I-\mathrm{SN}-q u i-\mathrm{SV}\right]$ :

1) contrainte sur l'existence, dans la construction, d'une seconde référence (éventuellement implicite seulement) au verbe avoir;

2) contrainte sur les temps verbaux (présent, imparfait, ou éventuellement temps composés correspondants);

3) contrainte sur le prédicat de la relative (prédicat obligatoirement spécifiant);

4) contrainte sur la négation (impossible) du verbe avoir.

Elles constatent, en observant leurs exemples, que ces contraintes ne s'appliquent pas de la même façon pour les différentes formes de la construction. Les exemples dans lesquels avoir est conjugué aux première et troisième personnes répondent de

12 Cf. les chapitres 1 à 3 de Furukawa, 2005 pour une démonstration de cette analyse. 
façon systématique aux contraintes 1), 2) et 3), mais offrent de nombreux exemples dérogeant à la contrainte 4). Au contraire, les exemples à la deuxième personne ne semblent pas soumis aux contraintes 1) et 2), et offrent très peu d'exemples dérogeant à la 4). Les auteurs appliquent l'examen des contraintes également à leurs exemples en [il y $a-\mathrm{SN}$ - relative prédicative], et observent que ces derniers montrent un comportement tout à fait semblable aux exemples à la deuxième personne. Elles proposent ainsi de dégager deux types de constructions : le type I [j'ai/il a - SN relative prédicative], illustré ici par (14) et (15), et le type II [tu as/il y a - SN - relative prédicative], illustré par (16) et (17).

(14) j'avais ma tante qui cherchait justement une euh une employée. (Lagae \& Rouget, 1998, p. 317)

(15) ils ont moins les magasins qui les attirent. (ibid.)

(16) tu as le gars qui regardait la table de montage seize. (ibid., p. 320)

(17) monsieur le président il y a la Suisse qui a envahi notre pays. (ibid., p. 321)

Elles proposent l'hypothèse que le type II représenterait un état plus avancé dans la désémantisation du verbe avoir, et plus haut dans le degré de grammaticalisation. Il est intéressant de noter que les auteurs semblent donc bien considérer les séquences en [tu as/vous avez] comme des variantes de celles en [il y a] ; le rapprochement entre les deux est là totalement explicite.

L'analyse syntaxique, quant à elle, n'est pas du tout centrale dans leur étude, et elle est donc très peu développée: les auteurs disent simplement considérer ces structures comme contenant une relative prédicative. Elles mentionnent un postulat de Léard (1992) faisant de certaines constructions en [il y a SN qui] une forme de proposition clivée, mais le rejettent aussitôt, considérant qu'une telle description peut être intéressante au niveau sémantique, mais «l'est moins sur le plan syntaxique » (ibid., p. 316).

1.1.4 Lambrecht s'est beaucoup intéressé aux structures en [il y $a-\mathrm{SN}-q u i-$ $\mathrm{SV}]$ et $\left[J^{\prime} A I-\mathrm{SN}-q u i-\mathrm{SV}\right]$, qu'il considère comme représentant un même type de construction; il propose une description très détaillée de leur comportement et de leurs fonctions dans son article de 2002. Ces structures forment pour lui le type le plus fréquent de ce qu'il appelle la «Construction relative présentative (CRP) » ${ }^{13}$ (Lambrecht, 2000, p. 49), construction qui a pour caractéristique de remplir une fonction discursive double : à la fois présenter une entité nouvelle et prédiquer une information nouvelle à propos de cette entité. Les cas avec avoir représentent ainsi une forme de CRP parmi d'autres : l'exemple (18) peut être mis en parallèle avec les exemples (19) à (22).

(18) J'ai eu mon beau-frère qui a fait Paris-Nice. (Lambrecht, 2000, 1(b))

(19) Il était une fois une belle princesse qui vivait dans un vieux château. (ibid., $1(\mathrm{a}))$

(20) Y a le téléphone qui sonne. (ibid., 1(c))

${ }^{13}$ En anglais : PRC, « Presentational Relative Construction », p. ex. Lambrecht, 2002, p. 171. 
(21) Je vois le facteur qui arrive. (ibid., 1(d))

(22) Voilà le facteur qui arrive. (ibid., 1(e))

La CRP est analysée comme une construction à prédication seconde : la première prédication est effectuée par le verbe (le verbe avoir pour les exemples qui nous concernent) et son objet (c'est la «proposition principale », Lambrecht, 2000, p. 50); la seconde intervient entre le sujet et le verbe de la relative. La relative est par ailleurs également «complément du verbe de la principale, dont la valence se trouve augmentée d'un terme » (ibid.). Dans cette construction, selon l'auteur, l'argument occupant syntaxiquement la place d'objet est, sémantiquement, un sujet. C'est le pronom relatif qui va porter la marque formelle du rôle sémantique du SN : il apparaitra le plus souvent sous la forme (sujet) qui.

Des contraintes lexicales pèsent par ailleurs sur la construction : seuls des verbes existentiels (et en première place le verbe avoir) ou des verbes de perception peuvent occuper la position de verbe «principal» (ibid.). Des contraintes existent également au niveau grammatical : « restrictions on the type of predicate, on negation, modality, and aspect, as well as on the morphosyntactic and prosodic form of the constituent denoting the presented entity » (Lambrecht, 2002, p. 174).

L'apparition des CRP est motivée pragmatiquement, par un principe que Lambrecht appelle PSRR, "Principe de la Séparation entre Référence et Relation» (Lambrecht 2000, p. 51) ${ }^{14}$. Cette contrainte cognitive universelle, présente en français parlé et dans de nombreuses autres langues,

s'exerce sur le cumul de la fonction présentative et de la fonction prédicative au sein d'une même structure argumentale, c'est-à-dire d'une même unité de traitement cognitive. Cette contrainte cognitive donne lieu à un principe d'expression selon lequel une nouvelle entité discursive se code de préférence dans une unité propositionnelle séparée. (Lambrecht, 2000, p. 51)

Les CRP respectent ce principe : le SN y a d'abord le statut d'objet/prédicat puis celui de sujet d'une prédication seconde. Un sujet lexical (dont le référent n'est pas actif à ce moment du discours) proposé directement en tête d'énoncé irait à l'encontre de ce principe. La CRP permet à ce SN d'être introduit par une structure présentative (il est 'objet'), puis de servir à une prédication. Les deux actions (présentation et prédication) sont réalisées dans une seule séquence, mais bien en deux étapes, grâce à la structure bipropositionnelle de la CRP.

Pour le cas plus spécifique des CRP en J'AI ou en il y a, Lambrecht (2002, p. 181) précise que le verbe avoir y figure dans son usage existentiel (correspondant à des emplois du verbe être dans d'autres langues), et que son sujet n'y a pas son rôle possessif: il est lié à une fonction locative, tandis que l'objet du verbe est lié à la fonction de thème. L'auteur propose un classement sophistiqué des CRP en avoir, selon deux ${ }^{15}$ paramètres fonctionnels : l'accessibilité (et le degré d'activation), pour

\footnotetext{
${ }^{14}$ En anglais : «Principle of the Separation of Reference and Relation » (Lambrecht, 1994, chap. 4 et 2002, p. 174).

${ }^{15}$ Lambrecht décrit en fait trois paramètres, mais dit lui-même que les deux derniers sont liés et se recoupent partiellement, et qu'il négligera donc généralement la distinction entre les deux. Il formule
} 
l'interlocuteur, du référent du $\mathrm{N}$ introduit; le caractère asserté ou au contraire présupposé du contenu de la relative. Il aboutit ainsi à trois sous-types de CRP en avoir : « existentiel », " clivé » et « énumératif » (cf. le Tableau 1).

Le sous-type énumératif est un peu à part, mais à l'intérieur des deux autres sous-types une nouvelle distinction apparait, puisqu'ils peuvent chacun remplir l'une des deux sous-fonctions pragmatiques suivantes :

1) introduire une entité nouvelle dans le discours, afin de la rendre susceptible d'une prédication ultérieure (dans ce cas l'entité introduite devient un topique par rapport à la relative qui suit) - c'est la fonction présentative en tant que telle ;

2) présenter une situation nouvelle, qui elle-même inclut bien sûr une entité nouvelle.

\begin{tabular}{|c|c|c|c|c|c|}
\hline \multicolumn{2}{|c|}{$\begin{array}{l}\text { CRP existentielles } \\
\text { entité nouvelle } \\
=\text { 'hearer-new' } \\
(<=>\text { SN indéfini) }\end{array}$} & \multirow{2}{*}{$\begin{array}{c}\text { CRP } \\
\text { existentielles } \\
\text { avec } \\
\text { « problème de } \\
\text { catégorisation " } \\
\text { Avec } \\
\text { quantifieur } \\
\text { dans le SN }\end{array}$} & \multicolumn{2}{|c|}{$\begin{array}{c}\text { CRP clivées } \\
\text { entité nouvelle = 'hearer- } \\
\text { old' } \\
\text { mais 'discourse-new' } \\
(<=>\text { SN défini })\end{array}$} & $\begin{array}{c}\text { CRP } \\
\text { énumératives }\end{array}$ \\
\hline $\begin{array}{l}\text { Statives } \\
\text { (entité } \\
\text { nouvelle) }\end{array}$ & $\begin{array}{l}\text { Événemen- } \\
\text { tielles } \\
\text { (situation } \\
\text { nouvelle) }\end{array}$ & & $\begin{array}{l}\text { Statives } \\
\text { (entité } \\
\text { nouvelle) }\end{array}$ & $\begin{array}{l}\text { Événemen- } \\
\text { tielles } \\
\text { (situation } \\
\text { nouvelle) }\end{array}$ & \\
\hline \multirow{2}{*}{$\begin{array}{l}\text { J'ai encore un } \\
\text { formulaire que } \\
\text { j'ai pas } \\
\text { elle a une dent } \\
\text { qui manque }\end{array}$} & $\begin{array}{l}\text { J'ai eu trois } \\
\text { personnes qui } \\
\text { sont venues se } \\
\text { plaindre }\end{array}$ & $\begin{array}{l}\text { Ily a beaucoup } \\
\text { d'Américains qui } \\
\text { approuvent la } \\
\text { violence }\end{array}$ & $\begin{array}{l}\text { Elle a son } \\
\text { père qui est } \\
\text { médecin }\end{array}$ & $\begin{array}{l}\text { Elle a sa } \\
\text { voiture qui est } \\
\text { chez le } \\
\text { mécanicien }\end{array}$ & $\begin{array}{l}\text { y a Beth qui veuty } \\
\text { aller, y a Jean- } \\
\text { Marc, y a moi... }\end{array}$ \\
\hline & & $\begin{array}{l}\text { "pas réellement } \\
\text { présentatives ", } \\
\text { clivées }\end{array}$ & & & $\begin{array}{l}\text { Relative } \\
\text { pragmatiquement } \\
\text { présupposée }\end{array}$ \\
\hline
\end{tabular}

Tbl. 1 - Les CRP en avoir (avoir personnel ou ily a), synthèse de Lambrecht, $2002^{16}$

Lambrecht aboutit ainsi à un classement en trois sous-types, dont deux sont encore subdivisés. On notera que, si certaines CRP en avoir sont qualifiées de « clivées », une seule et même analyse syntaxique s'applique à toutes : il s'agit toujours de prédication seconde (cf. le deuxième paragraphe de ce point, supra).

ainsi le troisième, non cité ci-dessus : « the pragmatic relation between the RC [=Relative Construction] denotatum and the global predication as FOCAL or TOPICAL » (Lambrecht, 2002, p. 182).

${ }^{16}$ Ce tableau vise à offrir une synthèse accessible de l'article de Lambrecht, 2002, assez complexe ; il n'est donc pas repris directement du texte de Lambrecht mais a été réalisé par mes soins: les éventuelles erreurs ou inexactitudes sont de mon fait. 


\subsection{Discussion des descriptions présentées, et nouvelles propositions}

Un élément me semble être à relever dans les quatre descriptions synthétisées plus haut: le statut du verbe avoir, et sa relation avec le $\mathrm{SN}$ qui le suit, est très peu problématisé. Du point de vue syntaxique, le verbe apparait comme constructeur; il n'y a que du point de vue sémantique que l'on relève parfois qu'il serait plus ou moins désémantisé.

Rothenberg signale à propos de l'un de ses exemples (J'avais ma petite maison qui ne se louait pas) que «avoir n'[y ]est pas l'équivalent lexical de posséder» (1979, p. 357); elle ne dit toutefois pas quel statut a le verbe dans cet exemple, et elle laisse ainsi entendre que dans les autres cas il a bien, justement, celui d'« équivalent lexical de posséder», soit un rôle de verbe constructeur. Furukawa (2005, chap. 1) parle quant à lui d'un avoir attributif plutôt que possessif, mais il n'en tire pas vraiment de conséquences au niveau de l'analyse syntaxique. Lambrecht, enfin, précise que le verbe avoir a ici une 《fonction existentielle» (« existential function», 2002, p. 180), et que son sujet n'a pas de rôle de possesseur - mais le SN qui suit avoir n'en semble pas moins constituer pour lui un objet direct du verbe.

L'interprétation en termes de prédication seconde/de relative prédicative, en effet, implique assez nécessairement que la relation entre avoir et le $\mathrm{SN}$ qui le suit soit de type "complément de verbe direct », et c'est la position que semblent défendre les auteurs évoqués ${ }^{17}$. On va voir qu'il y a là matière à discussion : examinons d'un peu plus près la question du rôle du verbe avoir dans la construction. Cette question étant centrale dans l'analyse, le fait de se tourner vers d'autres types d'explications va nous obliger à revoir complètement l'analyse de la construction.

1.2.1 Plusieurs auteurs ont relevé que l'on n'a pas affaire ici au verbe avoir " possessif », « verbe sémantiquement plein qui sélectionne deux arguments » (Tellier, 1992), ou verbe constructeur. Blanche-Benveniste l'a montré déjà dans son article de $1982^{18}$ : elle remarque que le verbe avoir n’a pas ici toutes ses possibilités de construction, et n'est donc pas un verbe de plein statut.

Par exemple, « il ne peut pas s’adjoindre facilement des compléments de rection comme 'sans peine', ou 'avec difficulté'» (Blanche-Benveniste, 1982, p. 94) :

(23) *j'ai eu sans peine ma collègue qui arrivait à deux heures (exemple modifié d'après (2), < CRFP, PRI-CLE-3)

Un exemple avec avoir constructeur peut, au contraire, s'adjoindre de tels compléments :

(24) eux ils ont eu sans peine le logiciel qu'on a développé (exemple modifié d'après (6), < CRFP, PRO_PSE)

Par ailleurs, le verbe avoir, ici, «n'est pas libre de ses temps, modalités et aspects; en particulier, on ne peut pas le mettre à l'impératif » (Blanche-Benveniste, 1982, p. 94),

\footnotetext{
17 On supposera ainsi que Lagae \& Rouget (1998) ont la même position.

${ }^{18}$ Dans cet article, elle examine diverses constructions traditionnellement décrites comme des subordonnées, et montre que dans le cas qui nous intéresse il ne s'agit pas de subordination : le verbe avoir n'est pas un verbe recteur standard, la relative n'en est pas vraiment une.
} 
alors que ces possibilités de conjugaison sont préservées dans un exemple comportant une « vraie » relative et un verbe avoir constructeur.

(25) *aie ta collègue qui arrive à deux heures (exemple modifié d'après (2), $<$ CRFP, PRI-CLE-3)

(26) ayez le logiciel qu'on a développé d'ici la semaine prochaine (exemple modifié d'après (6), < CRFP, PRO_PSE)

Cappeau (2005, p. 72) a également relevé qu'aucune proportionnalité ne pouvait être établie avec un pronom dans les structures en $[A V O I R-\mathrm{SN}-q u i-\mathrm{SV}]^{19}$, au contraire de ce qui se passe quand le verbe avoir construit un $\mathrm{SN}$ avec relative :

(27) *ma collègue qui arrive à deux heures, je l'ai (exemple modifié d'après (2), $<$ CRFP, PRI-CLE-3)

(28) le logiciel qu'on a développé, ils l'ont (exemple modifié d'après (6), < CRFP, PRO_PSE)

On voit ainsi qu'on n'a pas affaire dans nos constructions au verbe avoir qui construit un complément direct, comme avec un SN à relative adjective.

Certains auteurs ont par ailleurs montré que le type de construction en question n'est pas non plus semblable à ce qui se présente dans les structures avec relative attributive ou relative de perception. Comme le relèvent Lagae \& Rouget, suivant en cela l'idée de Rothenberg (1979, p. 370), alors que le SN tête d'une « relative attributive» peut être pronominalisé ((29)- (30)), ce n'est pas le cas avec les « relatives prédicatives » des structures en avoir ((31)-(32)) :

(29) il [un gorille] entend le bébé qui pleure (< Lagae \& Rouget, 1998, p. 313)

(30) il l'entend qui pleure (< Lagae \& Rouget, 1998, p. 314)

(31) ça va pas j’ai la petite qui est fatiguée (< Lagae \& Rouget, 1998, p. 313)

(32) *je l'ai qui est fatiguée (< Lagae \& Rouget, 1998, p. 314)

Selon elles, on ne peut donc pas considérer les structures en avoir et celles à relative de perception comme relevant de la même construction.

On doit conclure de tout cela que le verbe avoir a un statut tout à fait particulier dans la construction étudiée ; il n'entretient pas avec le SN qui le suit une relation de complémentation traditionnelle. Si l'on prend au sérieux les démonstrations qui précèdent, on doit aussi renoncer à l'analyse en termes de prédication seconde

19 À noter que Cappeau s'intéresse pour sa part aux exemples avec SN indéfini, et donne (2005, p. 72) les exemples suivants, en précisant que le dernier n'est pas mal formé, mais reçoit une interprétation très différente de l'énoncé initial, et ne lui est donc pas proportionnel :

$$
\begin{aligned}
& \text { j'ai une sœur qui vit aux Antilles / *je l'ai } \\
& \text { j'ai une sœur qui vit aux Antilles / ?j'en ai une } \\
& \text { une sœur qui vit aux Antilles, j'en ai une }
\end{aligned}
$$

Cf. ma partie 3.2.2 pour une discussion des différences entre structures à $\mathrm{SN}$ possessif et à $\mathrm{SN}$ indéfini. 
intervenant après une première prédication $[$ avoir $-\mathrm{SN}]$ : une telle première prédication n'existe pas.

1.2.2 Dès lors, quelle analyse proposer? On trouve des pistes de recherche intéressantes dans Léard (1992), et Berrendonner (2008), ainsi que dans une partie des analyses de Lambrecht (2002), qui seront donc exploitées ici.

Dans son livre de 1992, au détour de son étude approfondie des constructions en il y a, Léard effectue (dans une note de bas de page) un renvoi aux «structures possessives du type J'ai ma bagnole qui est en panne » (1992, p. 31). Il les rapproche alors explicitement « du clivage par ily a », illustré par les exemples Il n'y a qu'avec toi que je me sens en sécurité, et Ily a le chien qui court après un lapin.

Lambrecht adopte lui aussi le terme de "construction clivée», soit "structure syntaxiquement complexe mais sémantiquement simple» (2000, p. 51), pour qualifier certains des exemples qu'il étudie. Témoigne du clivage l'équivalence de ces structures avec une structure 'simple' correspondante : dans l'exemple suivant, le même contenu propositionnel peut en effet être exprimé «sous la forme canonique $\mathrm{SV}(\mathrm{O})$ » (ibid.) avec une valeur de vérité identique :

(33) J'ai eu mon beau-frère qui a fait Paris-Nice.

(34) Mon beau-frère a fait Paris-Nice. (exemple construit dans Lambrecht 2000, p. 51 et 2002, p. 174).

Berrendonner, enfin, donne une rapide description des structures en [ily $a-\mathrm{SN}$ - qui - SV] et [J'AI-SN - qui - SV] dans son article de $2008^{20}$. Pour lui (p. 288-289), dans ces constructions,

le verbe principal (y) avoir est un « auxiliaire de dispositif» (Blanche-Benveniste 1990), c'est-à-dire un verbe désémantisé faisant office de simple recteur grammatical, et permettant d'introduire en position de régime un $\mathrm{SN}$ support de prédication, plutôt que d'en faire un sujet. Dans ce dispositif, l'auxiliaire régit deux constituants à sa droite : un $\mathrm{SN}$, et une proposition enchâssée dont un argument pronominal est contrôlé par ce $\mathrm{SN}$, ce qui lui confère une forme analogue à celle d'une relative.

On n'est probablement pas très loin du concept de clivage ${ }^{21}$, mais la perspective est là un peu différente, envisagée cette fois dans les termes de la grammaire aixoise avec la notion de dispositif22.

${ }^{20} \mathrm{Il}$ y étudie l'alternance que/\#, notamment dans les structures en il y a et en J'AI : il y avait un mec qui faisait la queue / il y avait un mec \# il faisait la queue ; j'ai ma femme qui est alsacienne / alors j'ai un copain ici \# il rigolait. Pour une étude approfondie sur la même question, cf. Avanzi, 2012/sous presse, chap. 7 ; cf. aussi Willems \& Meulleman, 2010 (sur la même alternance mais uniquement dans les structures avec ily a).

${ }^{21}$ Le clivage est, dans la grammaire aixoise, une forme de dispositif syntaxique ; cf. infra le point 2.2.

22 À ma connaissance, Blanche-Benveniste n'a jamais parlé de « dispositif » pour qualifier la structure en [J'AI-SN-qui-SV]. Elle la rapproche en effet des structures en [ily $a-\mathrm{SN}-q u i-\mathrm{SV}]$, et fait alors de J'AI dans ces exemples "une sorte de version personnelle de il y a» (2010, p. 135); elle précise que, «dans ces tournures, [j’ai $\mathrm{N}$ qui] n'indique pas la possession mais la façon dont la personne est concernée par une propriété du nominal» (ibid., p. 136); elle ne parle toutefois pas (plus ?) explicitement de « dispositif ». 
«Clivage », « dispositif »: ces deux termes se recoupent-ils ? Et ces concepts peuvent-ils rendre compte de nos exemples, et cela plus avantageusement que "prédication seconde»? Je propose de discuter ces notions pour tenter d'apporter une réponse à ces questions.

\section{Les notions de " dispositif syntaxique " ou de " clivage " peuvent- elles s'appliquer à la construction étudiée?}

Pour beaucoup de linguistes, le terme de clivée s'applique uniquement aux structures avec extraction par c'est... quil que. Léard et Lambrecht, toutefois, comme on l'a vu, l'utilisent également pour décrire certains emplois de ily a ... qui et J'AI... qui. Le terme de dispositif, quant à lui, a été introduit il y a une trentaine d'années par BlancheBenveniste \& al. pour rendre compte des différentes organisations possibles d'une construction verbale. Ces deux notions se recoupent partiellement (la construction clivée est l'une des formes possibles des dispositifs syntaxiques); voyons successivement les spécificités de chacune, je discuterai ensuite de leur éventuelle application à la construction étudiée.

\subsection{La clivée}

Pour Riegel \& al. (2009[1994], p. 725), la phrase clivée est le résultat d'une extraction, "procédé emphatique qui associe une locution identifiante [...] et une relative pour extraire un constituant» : ce dernier " est extrait de la phrase et placé au début de celle-ci, encadré par c'est [...] et par le pronom relatif[23] qui ou que.»

L'extraction est «une opération de focalisation d'un constituant» (ibid., p. 726). Du point de vue sémantique, «l'information apportée par la phrase se dissocie en posé et présupposé », de la façon suivante (ibid.) :

- La séquence qui suit le relatif est présupposée : elle n'est pas affectée par la négation ni par la question. On peut nier l'élément extrait : Ce n'est pas Claire qui aime le chocolat; mais le présupposé (« quelqu'un aime le chocolat») n’est pas mis en cause.

- L'élément extrait est posé : c'est la seule information nouvelle apportée par la phrase clivée. Il s'identifie à la variable de la partie présupposée et la spécifie. Il s'oppose à un autre élément spécifique, qu'il exclut et que l'on peut expliciter : C'est Claire qui aime le chocolat, et non pas Régine. Avec la négation (Ce n'est pas Claire qui aime le chocolat), l'effet contrastif est encore plus appuyé : une valeur possible de la variable (Claire) est niée.

\footnotetext{
${ }^{23}$ Les auteurs parlent ici de relatif, mais on relèvera un peu plus loin une remarque bien intéressante à ce sujet :

Il est difficile, dans la plupart des cas, de traiter l'élément extrait comme un antécédent du relatif qui ou que qui le suit: la substitution est pratiquement impossible, notamment quand une subordonnée, un infinitif ou un complément circonstanciel sont extraits. Pour cette raison, on peut traiter que comme une conjonction. (Riegel \& al. 2009 [1994] : 728)
}

Cf. ici même le point 2.2 pour une discussion du statut de qu- dans la clivée. 
Il existe en français « deux séquences morphologiquement identiques » (BraultScappini, 2007, p. 53) qui recevront des interprétations et descriptions différentes : l'une est la clivée (ex. (35)), l'autre (ex. (36)) contient une véritable relative ${ }^{24}$.

(35) c'est lui qui l'a dit. (Brault- Scappini 2007, p. 53)

(36) c'est celui que j'ai vu. (ibid.)

Beaucoup d'énoncés sont, hors contexte, susceptibles des deux interprétations, ainsi par exemple (37) ci-dessous, interprété soit comme (38) soit comme (39).

(37) c'est le chat qui mange les souris. (< Brault- Scappini 2007, p. 53)

(38) c'est le chat (et pas le chien) qui mange les souris. (clivée/extraction) (ibid.)

(39) c'est celui qui mange les souris (relative). (ibid.)

Du point de vue de la syntaxe de la clivée, les éléments explicites (et consensuels) ne sont pas si fréquents. Lambrecht, dans sa définition de la clivée, donne des éléments d'analyse syntaxique :

Une Construction Clivée (anglais «cleft construction», Jespersen 193725) est une structure phrastique complexe composée d'une proposition principale à verbe copule et d'une proposition subordonnée de type relatif dont l'argument relativisé est coindexé avec le complément de la copule. Bi-phrastique sur le plan de la syntaxe, la clivée exprime une proposition logiquement simple, dans laquelle l'argument qui correspond au complément de la copule joue le rôle informationnel de focus. Cette proposition simple peut s'exprimer aussi dans une structure monophrastique sans changement dans les conditions de vérité. (Lambrecht, 2004, p. 21-22)

Mais plusieurs points me semblent discutables dans cette définition : les concepts de «phrase principale », de «complément de la copule», de «proposition subordonnée », et de «coindexation». On pourrait en fait envisager différemment les rapports entre les éléments de la structure. Quel statut accorder à c'est? Quel rapport entre c'est et le $\mathrm{SN}$ qui le suit? Quelle description pour ce qui ressemble à toute première vue à une 'subordonnée relative' ? Selon Blanche-Benveniste, « [i]l paraît assez vain de chercher à analyser les séquences c'est $N$ qu-comme si $N$ était le complément de c'est, suivi d'une relative» (2002, p. 109), et c'est donc un autre type de description qui sera proposé dans la grammaire aixoise. Je propose d'examiner cela dans la section suivante, puisque la clivée représente une forme de dispositif syntaxique.

\footnotetext{
24 Rouget \& Salze (1986) montrent qu'il existe en fait quatre types de structures différentes en c'est... $q u-:$ en plus de la clivée et de la structure à relative (déterminative), elles identifient aussi une structure à «greffe», avec une construction verbale détachée (c'est les Sibylles que Michel-Ange les peignait...) et une structure avec c'est + "groupe verbo-nominal " (la corrida c'est le matador qui s'amuse avec le taureau; [la table inondée / ø] c'est cette sacrée casserole qu'on en fout partout). Elles mettent en évidence les caractéristiques syntaxiques de chacune des structures, et en analysent des exemples issus de corpus oraux.

25 Jespersen, O., 1937, Analytic Syntax, London, Allen \& Unwin.
} 


\subsection{Les dispositifs}

2.2.1. Blanche-Benveniste ( 2000 : 158$)$ donne la définition ${ }^{26}$ suivante des dispositifs syntaxiques :

organisations des constructions verbales, existant pour à peu près tous les verbes, correspondant à différentes formes de macrosyntaxe et différentes répartitions de l'information: dispositif direct, elle veut des pommes; dispositif à antéposition, des pommes, elle veut; dispositif clivé, c'est des pommes qu'elle veut; dispositif pseudo-clivé, ce qu'elle veut, c'est des pommes.

On voit que la clivée en c'est... quil que examinée ci-dessus constitue ainsi l'un des dispositifs syntaxiques possibles en français. Blanche-Benveniste nous donne plusieurs éléments d'analyse proprement syntaxique pour la description de la clivée en tant que dispositif : je les relève ci-après.

«Les clivées $[\ldots]$ ont pour effet de séparer une construction verbale [...] en deux parties : l'une où se trouve un constituant de la construction verbale (à l'exclusion du verbe), $[\ldots]$ une autre partie où se trouve tout le reste de la construction. ${ }^{27}$ (Blanche-Benveniste, 2002, p. 103-104) Des contraintes existent sur l'opération (ibid., p. 105-106) :

(i) L'extraction ne peut jamais s'opérer sur le verbe, et ne peut pas s'opérer si le verbe n'est accompagné que de clitiques ${ }^{28}$ : le constituant sur lequel elle agit doit avoir un minimum d'autonomie.

(ii) Seul un constituant régi par le verbe peut être extrait, les «compléments de phrase » ou les subordonnées en puisque ou en si sont exclus de l'opération.

Et une particularité remarquable lui est associée (ibid., p. 106-107) : l'opération permet d'ajouter à l'énoncé une modalité, indépendante de celle du verbe 'principal', par exemple une seconde modalité négative, portant sur le complément, dans (41) (l'exemple (42) montre que la seconde modalité ne peut être appliquée sans l'opération de clivage). Les emplois avec une modalité négative portant sur le constituant clivé2 ${ }^{29}$ sont particulièrement fréquents à l'oral selon Blanche-Benveniste (cf. encore (43)) ; une modalité interrogative est également possible (cf. (44)).

(40) Paul ne voulait plus parler à ses amis. (Blanche-Benveniste, 2002, p. 106)

(41) ce n'est pas à ses amis que Paul ne voulait plus parler, c'est à ses collègues. (Blanche-Benveniste, 2002, p. 107)

(42) *Paul ne voulait plus parler pas à ses amis. (ibid.)

\footnotetext{
${ }^{26}$ Cf. aussi des éléments de définition du dispositif syntaxique dans l'article de Sabio ici même, point 1.

${ }^{27}$ Blanche-Benveniste applique cette caractéristique aux clivées et aux pseudo-clivées.

${ }^{28}$ Elle est possible avec les formes toniques des pronoms.

${ }^{29}$ Cf. ici même l'article de Sabio sur les séquences en c'est pas parce que... que... : il montre que ces constructions, quoique formellement proches d'une clivée avec modalité négative, ne peuvent en fait pas être décrites de la même façon comme des dispositifs clivés.
} 
(43) c'est pas pour ça que je crie, c'est pas parce que je suis sourd. (ibid.)

(44) Est-ce à ses amis que Paul ne voulait plus parler, ou à ses collègues. (ibid.)

Blanche-Benveniste (2001) propose un examen détaillé du "verbe c'est» dans la clivée. Elle met en évidence ${ }^{30}$ les caractéristiques suivantes :

(i) Il ne sélectionne pas ses valences : c'est l'autre verbe de la séquence qui est responsable du choix des compléments.

(ii) Il a ses propres modalités.

(iii) Il n'est pas support de clitiques.

(iv) Il ne s'accompagne que très rarement d'un auxiliaire.

(v) Il n'est pas très libre dans les variations de temps, et pratiquement pas dans les variations de personnes: il est le plus souvent à la $3^{e}$ personne du singulier, et parfois à la $3^{\mathrm{e}}$ personne du pluriel.

Ses propriétés verbales sont ainsi tout à fait limitées, et ne correspondent pas à celles d'un verbe de plein statut. Pour elle, le verbe c'est doit être considéré « comme un auxiliaire, au sens de 'verbe mis au service d'un autre', ayant un assez faible degré de verbalité » (2001, 93) ; il est « seulement le support matériel de l'élément clivé » (2002, 110).

L'élément qu-, quant à lui, ne peut vraisemblablement plus être considéré comme un pronom relatif ${ }^{31}$. Blanche-Benveniste propose simplement de le traiter «comme une particule assurant la délimitation du constituant » (2002, p. 110).

Finalement, « [ces] deux éléments, c'est et qu-, ont perdu une partie des caractéristiques de la catégorie verbale et pronominale, et ils sont devenus des outils grammaticaux » (ibid.).

Une telle description a l'avantage d'apporter des réponses convaincantes aux diverses questions soulevées par l'analyse de la clivée. C'est donc cette description qui sera retenue ici : la construction clivée en c'est... qu- est à considérer comme un dispositif syntaxique ; l'élément c'est y est 'peu verbal' ; le SN n'est pas son complément direct.

2.2.2 Les structures en [ily $a-\mathrm{SN}-q u i-\mathrm{SV}]$ constituent-elles également, dans le modèle aixois, un dispositif syntaxique particulier? Il faut se poser cette question, puisque plusieurs auteurs semblent les considérer comme telles ${ }^{32}$, et que je proposerai

30 Je ne reprends ici que les conclusions de sa démonstration : pour le développement complet cf. Blanche-Benveniste (2001, p. 88-93).

31 Il ne s'agit en effet plus là d'une véritable «relative», ce qui est rendu plus évident par des exemples dans lesquels «l'élément clivé n'est pas un nom [...]: C'est maintenant que je le vois» (Blanche-Benveniste 2002, p. 109). Cf. la note 23 ci-avant.

32 Cf. 1.2.2 : Berrendonner, 2008 ; dans d'autres perspectives Léard, 1992 et Lambrecht, 2000 et 2002 les considèrent comme des clivées, elles-mêmes considérées par la grammaire aixoise comme une forme de dispositif. Pour les trois auteurs cités, les constructions en [il y $a-\mathrm{SN}-q u i-\mathrm{SV}]$ et en $\left[J^{\prime} A I-\mathrm{SN}-q u i-\mathrm{SV}\right]$ sont analysées de façon similaire. 
moi aussi cette analyse. Les constructions en il y $a$ sont très diverses, en fait, et donc soumises à différentes descriptions dans la grammaire aixoise. Voyons les principaux cas, pour arriver finalement à celui qui nous intéresse.

En toute première approche, il faut déjà différencier les constructions dans lesquelles il y a est un verbe recteur ${ }^{33}$, plein, qui « situe l'existence d'un élément dans l'espace ou le temps» (Blanche-Benveniste 2010, p. 133) ${ }^{34}$, de toutes celles dans lesquelles il y a n'est pas un verbe de plein statut mais un «support à d'autres verbes » (ibid.). On peut ensuite mettre en évidence différents emplois d'il y a dans cette seconde catégorie (il y a non recteur, non plein) ${ }^{35}$, dont certains sont considérés comme des dispositifs.

Le il (n')y a (que) servant à la restriction est considéré comme l'auxiliaire d'un véritable dispositif syntaxique, qui opère aussi, comme la clivée, une forme de focalisation : il « est une sorte d'auxiliaire utilisé pour placer la restriction sur le sujet» (Blanche-Benveniste 2002, p. 108), cf. (45) et (46). Et, comme dans la clivée, le SN n'est pas dans sa valence, mais dans celle du 'second' verbe.

(45) le docteur ne le sait pas. (Blanche-Benveniste 2002, p. 108)

(46) Il n'y a que le docteur qui ne le sait pas. (ibid.)

Les structures en $[i l$ y $a-\mathrm{SN}-q u i-\mathrm{SV}]$ autres que la restriction sont étudiées principalement dans leurs formes avec $S N$ indéfini, et dans ce cadre elles reçoivent dans certains cas ${ }^{36}$ l'appellation de dispositif: Blanche-Benveniste (2000, p. 93) les considère comme des «dispositifs auxiliaires de la détermination nominale». Elles sont fréquemment utilisées en français parlé pour "encadrer» (ibid.) un sujet indéfini, type de sujet que l'oral réprouve en tout début d'énoncé : à l'oral on semble préférer (47) à (48).

(47) ily a peu de gens qui font quelque chose. (Blanche-Benveniste, 2010, p. 93)

(48) peu de gens font quelque chose. (ibid.)

En revanche, la structure en [il y a-SN - qui - SV] avec $S N$ défini ou possessif ne semble (curieusement) pas être décrite comme un dispositif. A ma connaissance, elle n'est pas souvent évoquée dans les écrits des chercheurs aixois, mais elle est mentionnée dans Blanche-Benveniste (2010), sous la rubrique traitant de il y a (p. 133135). Elle est considérée là (ex. (49) et (50) $)^{37}$ comme un emploi de ily a en tant que

\footnotetext{
33 J'utilise ici «recteur» au sens de « recteur fort», et comme synonyme de «constructeur»: ces termes indiquent que le verbe régit un ou des compléments, en tant que verbe de plein statut. Pour une discussion de ces notions cf. par exemple Blanche-Benveniste 1990, chap. II.

34 Elle donne, entre autres, l'exemple suivant d'emploi de plein statut de il y a: il y a une île entre le Japon et la Chine (Blanche-Benveniste 2010, p. 133).

${ }^{35}$ Le il y a «instrument spécifique de mesure temporelle, à la façon d'une préposition », ex. quand je suis arrivé il y a quatre ans (Blanche-Benveniste 2010, p. 134), ne sera pas traité ici puisqu'il n'entre pas dans le schéma morphosyntaxique qui nous intéresse.

36 Voir aussi la notion de «stabilisateur » développée dans l'article de Cappeau \& Deulofeu (2001).

${ }^{37}$ Ces exemples se ressemblent beaucoup, le contexte manque pour voir comment ils se sont succédé...
} 
verbe de plein statut, donc dans la première catégorie évoquée ci-dessus (verbe plein et non pas verbe support). Dans les exemples donnés, le verbe il y $a$ « intervient à ce titre [= de verbe plein] dans la relation de procès en séries » (ibid., p. 133).

(49) il y a la fille qui arrive - elle met encore sa voiture juste devant (BlancheBenveniste, 2010, p. 133)

(50) il y a son père qui arrive il met encore sa voiture (ibid.)

Les exemples en il y a avec déterminant défini dans le SN semblent donc ne pas être considérés comme des dispositifs syntaxiques chez Blanche-Benveniste, mais comme des usages d'il y $a$ en tant que verbe plein. De tels exemples (avec dét. défini) sont toutefois très rarement mentionnés dans les travaux aixois, les chercheurs concernés s'étant plutôt intéressés aux cas avec déterminant indéfini dans le SN.

Ici, il y a ne sera pas considéré comme un verbe plein dans ces exemples : le statut d'il y a y parait ${ }^{38}$ similaire à celui de $J$ 'AI dans les exemples prototypiques de la construction illustrée en (1)-(5), et on a vu (1.2.1 et 1.2.2) que, selon certains chercheurs, il est pertinent d'en rejeter la description en termes de verbe constructeur (cf. aussi la discussion qui suit, 2.3, pour le développement d'une analyse alternative). Je proposerai de considérer que ces constructions en AVOIR aussi bien personnelles qu'impersonnelles (il y a) ne présentent pas un verbe avoir de plein statut; elles sont similaires sur ce point, et seront donc traitées en parallèle ci-après.

\subsection{Discussion}

Que deviennent les principaux éléments vus ci-dessus (2.1 et 2.2.1), qui entrent dans la définition retenue de la clivée, si on tente de les appliquer pour caractériser les constructions en [AVOIR - SN - qui - SV] ? C'est ce que je propose d'examiner maintenant: je prendrai ces éléments l'un après l'autre, dans leur ordre d'apparition dans les sections évoquées, pour voir s'ils conviennent aussi bien aux constructions en J'AI et en il y a qu'à la clivée en c'est. On verra ainsi si l'on peut considérer toutes ces constructions comme une même forme de clivage, ou si, à défaut de pouvoir parler de clivées pour les trois, on peut au moins appliquer à toutes l'hyperonyme concerné, et considérer aussi les constructions en AVOIR comme une (autre) forme de dispositif syntaxique.

2.3.1 La structure en [AVOIR - SN - qui - SV] présente-t-elle, comme la clivée en c'est... qu-, une extraction, ou plutôt une séparation d'une construction verbale en deux parties (dans les termes de Blanche-Benveniste) ? C'est bien ce que l'on observe dans mes exemples, si l'on est d'accord de ne pas voir le SN comme un complément du verbe avoir - mais plutôt comme un élément sélectionné par le second verbe (cf. point 1.2.1). Ainsi dans l'exemple (51) ma copine n'est pas le complément de j'ai, mais le sujet de habite. L'élément j'ai sert de support pour cette extraction, cette organisation particulière de la construction verbale.

(51) j'ai ma copine qui habite à Paris (CRFP, PRI-BRI-1)

38 Cf. Conti, en cours pour une démonstration de cela. 
Il semble que les fonctions des constituants susceptibles de subir cette extraction soient toutefois moins variées que dans la clivée en c'est... qu- : l'extraction ne parait pouvoir porter que sur l'élément sujet ${ }^{39}$.

2.3.2 La clivée en c'est... qu- est toujours décrite comme une opération de focalisation; le SN y représente l'élément focus, alors que le segment à forme de relative marque par définition une information présupposée. Le statut de ce segment est bien différent dans nos exemples en AVOIR : le SV (à forme de relative) apporte toujours une information nouvelle et est donc dans tous les cas rhématique ${ }^{40}$ (par définition). ${ }^{41}$ Sur ce point, les constructions en $A V O I R$ ne correspondent donc pas à la clivée en c'est.

2.3.3 On a observé que la clivée en c'est... qu- pouvait être mise en parallèle avec une autre séquence morphologiquement identique, constituée d'un verbe c'est suivi d'un SN contenant une relative adjective (cf. exemples (35) à (39)). Ceci n'est probablement pas un critère définitoire de la clivée, mais il me parait intéressant de constater que mes exemples en AVOIR offrent la même possibilité : comme on l'a vu tout au début du point 1, la construction en AVOIR correspond à un schéma auquel répondent également des séquences en AVOIR - $\mathrm{SN}$ - relative adjective (cf. exemple (6)).

On ira même plus loin : un même énoncé peut parfois, hors contexte, recevoir les deux interprétations.

\section{(52) j’ai ma mère qui s'en occupe (CRFP, PRI_BAY_1)}

L'exemple (52) aurait pu être analysé comme une illustration de la construction en AVOIR étudiée ici (avec SV rhématique). Or son contexte d'apparition montre que cet énoncé est précédé d'une question portant sur le fait d'avoir ou pas quelqu'un qui peut garder ses enfants : on a donc certainement plutôt affaire ici à un cas de $\mathrm{SN}$ régi par le verbe avoir suivi d'une relative non rhématique (cf. l'exemple contextualisé au

${ }^{39}$ Blanche-Benveniste propose un exemple avec extraction du complément direct du verbe : moi j'ai encore un formulaire que j'ai pas (1982, p. 95). J'ai peu d'exemples de ce type, et j'attends d'en avoir davantage avant d'en proposer une explication.

40 Léard (1992, p. 52-53) montre, à côté des cas les plus fréquents avec SV rhématique, un seul type de clivage en il y a où le SV est thématique voire présupposé; il l'illustre notamment par l'exemple suivant :

A — Je me demande ce qui sera le mieux pour les bagages.

$\mathrm{B}$ - Il y a le sac qui est pratique

Lambrecht propose lui aussi, en marge des cas où la relative est rhématique, un type de CRP en avoir avec relative pragmatiquement présupposée: la CRP énumérative (cf. Tbl. 1 en section 1.1.4). Ces deux derniers exemples n'illustrent toutefois pas le même type de construction que celui qui est étudié ici: dans ces deux exemples, on a affaire à un il y a régissant un $\mathrm{SN}$ avec une relative appositive rappelant de l'information d'arrière-plan. Au contraire, dans la structure à l'étude ici, le SV ne rappelle jamais une information d'arrière-plan, il est toujours rhématique.

${ }^{41}$ Léard (1992, p. 34) propose d'analyser la plupart des clivées en il y a comme du 'tout rhématique' : SN rhématique, SV rhématique (à part pour le cas vu à la note précédente). Il renvoie là au concept de «jugement thétique» de Kuroda (Kuroda, S. Y., M.-L. Beffa et M. Borel, 1973, «Le jugement catégorique et le jugement thétique : exemples tirés de la syntaxe japonaise », Langages, n³0, p. 81 110). Lambrecht, quant à lui, dans la structure qu'il considère comme biphrastique, voit le SN comme un focus dans la première proposition, qui devient un topique dans la seconde, où il est sujet du prédicat (cf. par exemple 2000, p. 51). 
point 3.2.1). La même forme est susceptible des deux interprétations ; c'est le contexte, et plus particulièrement l'analyse informationnelle du contexte précédent, qui permet de trancher. Sur ce point les constructions en AVOIR rejoignent une des particularités de la clivée en c'est: la double interprétation possible de certains exemples (cf. 2.1 supra).

2.3.4 Les structures en AVOIR permettraient-elles, comme la clivée en c'est, de faire porter sur un constituant des modalités supplémentaires à celles qui sont attachées à la construction verbale ? Cela ne semble pas être le cas, et l'on peut probablement mettre ce fait en relation avec la différence observée au point 2.3.2: notre construction en $A V O I R$ ne sert pas à la focalisation d'un constituant, ne permet pas comme elle la mise en évidence d'un contraste. Des exemples comme (41) et (43) ci-dessus n'ont ainsi probablement pas d'équivalent dans la construction en AVOIR, et ceci du fait que la structure informationnelle de cette dernière ne le favorise pas (pas de focalisation sur un seul constituant avec ensuite SV présupposé: toute la construction verbale est rhématique ici, cf. 2.3.2). Les constructions en AVOIR ne semblent donc pas permettre les mêmes opérations que la clivée en c'est.

2.3.5 Tout comme c'est dans la clivée, AVOIR dans notre construction n'est pas un verbe de plein statut. Il ne sélectionne pas de compléments, n'est pas totalement libre dans sa conjugaison (cf. 1.2.1). Sur ce point, les différentes constructions se ressemblent.

2.3.6 Enfin, comme dans la clivée en c'est, le fait de ne plus parler de véritable proposition relative incite à voir l'élément qu- non pas comme un pronom relatif, mais comme une simple particule nécessaire à la construction, délimitant le constituant extrait. On n'a toutefois pas ici de contextes rendant évident ce fait, comme c'est le cas avec la clivée en c'est ${ }^{42}$ :

(53) C'est demain que le concours aura lieu (Riegel \& al. 2009[1994], p. 727).

2.3.7 Au final, quelle description doit-on retenir pour nos constructions en AVOIR ? Elles semblent se distinguer sur plusieurs points des clivées en c'est, et on gagnerait donc probablement à garder l'appellation de clivée uniquement pour ces dernières, dont les propriétés syntaxiques s'accompagnent d'une claire opération de focalisation sur un constituant extrait. Comme les constructions en AVOIR ne remplissent pas toutes les conditions définitoires, elles ne seront finalement pas considérées ici comme des constructions clivées (au sens strict vu ci-dessus).

En revanche, on devrait considérer que les constructions en AVOIR examinées ici, aussi bien celles en avoir personnel que celles en il y a, peuvent être décrites comme des formes particulières d'organisation d'une construction verbale (construction verbale dont le verbe apparait alors après l'élément qui dans nos séquences). Le statut particulier du verbe c'est (mis en évidence dans la description de la construction clivée en tant que dispositif) se retrouve dans les constructions en avoir, et de ce fait on y retrouve aussi le caractère non « relatif » de l'élément qu-, à voir plutôt comme un outil grammatical. On peut ainsi considérer qu'un constituant de la construction verbale est extrait et placé entre AVOIR et qui; la même construction verbale pourrait être

${ }^{42}$ Cf. les notes 23 et 31 ci-avant. 
présentée sous la forme d'un dispositif direct. Dans les termes de la grammaire aixoise, on devrait ainsi pouvoir appliquer la notion de dispositif syntaxique à ces constructions c'est en tous les cas la description que j'en propose ici.

\section{Entre syntaxe et discours...}

\subsection{Dépasser le cadre syntaxique}

J'espère avoir montré ci-dessus que le 'verbe' AVOIR, sous une forme personnelle ou sous sa forme impersonnelle il y a, peut être utilisé en français pour organiser de façon originale une construction verbale. J'aimerais en effet envisager l'hypothèse que l'on puisse, dans les cas décrits ici ${ }^{43}$, considérer les trois énoncés en (54)-(56) comme trois configurations différentes d'une même construction verbale, trois dispositifs syntaxiques $^{44}$.

(54) j'ai ma copine qui habite à Paris (exemple original, < CRFP, PRI-BRI-1)

(55) il y a ma copine qui habite à Paris (exemple modifié)

(56) ma copine habite à Paris (exemple modifié)

Il est nécessaire ici de dépasser le cadre syntaxique de l'analyse : pour compléter la description, on devra faire appel à des éléments d'analyse textuelle, au moins à deux titres.

(i) L'analyse proprement syntaxique ne rend pas compte des raisons de la sélection par le locuteur de l'une ou l'autre forme, en d'autres termes elle évacue l'étude du rôle de avoir, employé sous ses formes personnelles ou impersonnelle (il y a). L'observation des conditions de répartition de ces différents dispositifs dans le discours, et des divers rendements de ces constructions, doit apporter des éléments indispensables à la description, mais hors d'atteinte dans le cadre d'une analyse proprement syntaxique. Une telle étude dépasse le champ de cet article: ce point ne pourra pas être développé ici.

(ii) Le second point nous amène véritablement à l'interface entre syntaxe et discours: l'analyse syntaxique retenue est toujours dépendante du contexte d'apparition de la construction, de l'état de l'information partagée au moment de l'énonciation. J'aimerais maintenant illustrer ce constat.

\footnotetext{
43 Une description fine des cas pouvant se soumettre à une telle analyse (ou en d'autres termes la mise en évidence des contraintes précises pesant sur la construction en question) reste à faire : cf. plus loin le point 3.2 pour quelques premiers éléments, et cf. Conti, en cours pour une description plus complète (et pour un développement de l'analyse en termes de dispositifs syntaxiques).

44 J'aimerais signaler ce fait amusant que j'ai dans mes corpus deux exemples qui pourraient illustrer mes propos de façon très... authentique : (il) y a ton sac qui est tombé et tu as ton sac qui a cbu. Ces énoncés ont été prononcés (devant un 'même' évènement) autour de moi par deux locuteurs différents à quelques années d'intervalle.
} 


\subsection{Une analyse syntaxique en contexte}

3.2.1 Voyons un exemple déjà évoqué, mais cette fois dans un contexte un peu plus large.

(57) j’ai ma copine qui habite à Paris [CRFP, PRI_BRI_]

L1 c'est la voie donc + que j'ai j'ai que j'ai pris $\{$ sic $\}$ là pour mes études + si si je suis prof d'E.P.S. je suis sûr à quatre-vingt-dix pour cent que je vais être euh lancé euh sur Paris ou < région parisienne

L2 * banlieue parisienne $>$

L1 et je vais être étouffé déjà que j'ai ma copine qui habite à Paris + enfin qui à un appartement à Paris et + euh quand j'y monte je je respire plus quoi

L2 hum hum

Ici, l'énoncé est 'tout rhématique', et correspond bien à la structure décrite - il en est même probablement un exemple assez 'prototypique'. Voyons maintenant un cas (déjà évoqué également, cf. 2.3.3) qui révèle une analyse sensiblement différente.

(58) j’ai ma mère qui s'en occupe [CRFP, PRI_BAY_1]

L1 ça vous a changé le rythme de vie

L2 oui aussi oui $<$ oui

L1 pourquoi $>$ vous faisiez quoi avant que vous ne pouvez plus faire

L2 moins de sorties mais bon ça pff ça fait rien de toute façon hein + moins de sorties après c'est sûr que pour garder pour le travail faut s'arranger +

L1 hum hum

L2 pour la faire garder si on veut travailler avec un enfant c'est vrai que c'est pas facile si on (n') a pas quelqu'un /de, dans/ la famille qui puisse euh qui puisse euh la garder quoi

L1 vous avez

L2 oui j'ai ma mère qui s'en occupe + heureusement $\{$ rire $\}+$ non heureusement je sais pas la chance que j'ai euh moi je sais qu'elle est bien + avec elle

L1 hum hum

L2 c'est vrai que j'aurais peur de la donner à un étranger quoi par contre là ça serait dur

Là, au contraire de l'exemple précédent, l'état de l'information partagée ainsi que l'intonation (légère pause après mère) montrent qu'on n'a pas affaire à cette structure : il s'agit plutôt d'un SN régi par le verbe avoir, suivi d'une relative non rhématique. On ne peut appliquer ici l'analyse en dispositif, alors que cet exemple suit exactement le même schéma de surface que le précédent.

On se rappellera qu'on a observé ce phénomène également pour les clivées en c'est; la question se pose peut-être de savoir s'il n'est pas plus fréquent qu'on le pense généralement.

3.2.2 Deux séquences morphologiquement identiques peuvent donc recevoir deux analyses différentes ; à l'inverse, des éléments morphologiques différents peuvent 
apparaitre dans la structure étudiée, qui ne semble pas figée dans un seul schéma : le SN contient très souvent un déterminant possessif, mais il peut aussi présenter un article défini, ou même un déterminant indéfini. Les exemples (59)-(61) peuvent être considérés comme illustrant la construction décrite ici.

(59) J'ai le 01.72.34.93.27 qui me contacte régulièrement, au départ je ne décrochais pas, mais j'ai été curieuse de savoir qui m'appelais sans arrêt et quand je décroche, je n'ai strictement pas de réponse. Si quelqu'un peut m'éclairer sur ce numéro bizard, merci (Internet, sic)

(60) ben moi j'ai un gars qui me raconte l'inverse hein en ce moment (oral)

(61) et moi il y a une chose qui m'a surpris c'est que euh + euh au cours d'une discussion comme ça dans la rue euh auprès des petites échoppes où on peut manger boire euh + euh j'ai des des des des je des des des Noirs qui m'ont dit écoutez ne vous faites pas de soucis euh vous serez partis d'ici la fin de la semaine et il s'est trouvé ça s'est révélé exact par la suite (CRFP, PRI_AIX_)

Au contraire, les constructions suivant le même schéma mais possédant un SN avec un article défini et un $\mathrm{N}$ dont le référent est en rapport de partie à tout avec le référent du sujet d'avoir ne peuvent pas recevoir la même description en termes de dispositif : ils ne présentent pas la même équivalence relative que les énoncés (54)-(56).

(62) j'ai le nez qui pique (oral)

(63) et puis depuis ce moment-là j'ai l'oreille qui siffle (oral, radio)

Un critère sémantique permet d'isoler ces exemples : la relation partie-tout. Un même genre de critère permettrait-il d'exclure également certains exemples avec déterminant indéfini, de les différencier des exemples (60)-(61) ? Dans (64), on peut relever la présence d'un $\mathrm{N}$ relationnel.

(64) chères amies

J'ai une amie qui a besoin de vous pour son film.. je vous transfère le mail.. à tout bientôt

bec

(courriel)

Cette caractéristique sémantique du $\mathrm{N}$ semble pouvoir rétablir le verbe avoir dans ses capacités de construction verbale, et l'analyse en dispositif syntaxique en devient bien moins évidente. On se trouve peut-être là dans un cas de métanalyse (Béguelin, 2000, 2002, 2004): la structure serait soumise à double description syntaxique possible (dispositif ou verbe avoir recteur).

En fait, les exemples avec SN indéfini ouvrent tout un nouveau champ d'investigation, qui dépasse le cadre de cet article ${ }^{45}$. La question du type de SN (types

${ }^{45}$ Cf. notamment Conti (2004), Cappeau (2005) et Conti (en cours) pour des pistes d'analyse des structures en $\left[J^{\prime} A I-\mathrm{SN}-q u i\right]$ avec SN indéfini ; Cappeau \& Deulofeu (2001), Cappeau \& Deulofeu (2006) pour celles en [ily $a-\mathrm{SN}-q u i]$. 
de déterminations) qui peut entrer dans la structure ne peut ainsi être réglée une fois pour toutes, hors contexte ; la nécessité pour l'analyse d'un va-et-vient constant entre la (morpho-)syntaxe et le discours se confirme.

\section{Conclusion}

Beaucoup d'éléments d'analyse ont dû être laissés de côté ici : une description complète des emplois possibles des constructions en [AVOIR $-\mathrm{SN}-q u i-\mathrm{SV}]$ ne peut tenir en quelques pages. Je me suis donc concentrée sur la syntaxe, et j’ai tenté de montrer qu'une analyse approfondie des structures étudiées ne pouvait manquer de mettre en évidence des particularités qui peinent à se laisser expliquer uniquement au moyen des outils de la grammaire traditionnelle (subordination, prédication seconde, etc.). Le statut du verbe avoir ici, la relation qu'il établit avec les autres éléments de la construction, obligent à remettre en question les analyses en termes de prédication seconde. Certains modèles élaborés en tenant compte de l'étude du français parlé (modèles de micro-/macrosyntaxe) offrent alors des outils intéressants. L'analyse que je propose, en dispositif syntaxique, fait entrer la structure en [AVOIR - SN - qui - SV] dans un paradigme bien établi de constructions syntaxiques très fréquentes en français oral, dont l'étude me semble documenter avantageusement la description du français contemporain.

J'ai par ailleurs insisté sur la nécessité d'associer les niveaux d'analyse : la description de la construction étudiée doit se faire dans une interaction constante entre syntaxe et discours. L'analyse syntaxique ne peut se faire hors contexte; l'analyse discursive quant à elle doit partir d'une description syntaxique fine, qui permet d'éviter de trop grandes généralisations dans l'étude des emplois des constructions en discours, en encourageant à ne pas considérer toutes les instanciations d'un «schéma général » comme des illustrations d'une seule et même construction. Il faut alors absolument observer de grands corpus, pour étudier d'aussi près que possible l'importante variation à laquelle on est confronté ici : variations morphosyntaxiques possibles de la construction décrite, d'une part ; variation des descriptions qu'il faudra donner des diverses illustrations du « schéma général » évoqué en début d'article d'autre part. On se trouve ainsi devant la tâche lourde mais passionnante de décrire une langue dans toute sa complexité. 
Virginie Conti

\section{BIBLIOGRAPHIE}

AvanzI, M. (2012/sous presse), L'interface prosodie/syntaxe en français. Dislocations, incises et asyndètes, Bruxelles, Peter Lang, collection Gramm-R.

BÉGUELIN, M.-J. (2000), «Des clauses impersonnelles aux constituants phrastiques : quelques axes de grammaticalisation ", in Seriot, P. \& A. Berrendonner (eds.), Le paradoxe du sujet. Les propositions impersonnelles dans les langues slaves et romanes, Cabiers de l'ILSL n¹2, Lausanne, p. 25-41.

BÉGUELIN, M.-J. (2002), « Routines macro-syntaxiques et grammaticalisation : le cas des clauses en n'importe ", in Leth Andersen, A. \& H. Nølke (eds.), Macro-syntaxe et macro-sémantique. Actes du Colloque international d'Aarbus, 17-19 mai 2001, Berne, Peter Lang, coll. Sciences pour la communication, p. 43-69.

BÉGUELIN, M.-J. (2004), «Coalescences et dégroupages syntaxiques : réflexions sur les conditions pragmatiques et sémantiques des grammaticalisations », in Auchlin, A. et al. (eds.), Structures et discours. Mélanges offerts à Eddy Roulet, Québec, Éditions Nota Bene, p. 139-150.

BERRENDONNER, A. (2008), «L'alternance que/\#. Subordination sans marqueur ou structure périodique? » in Van Raemdonck, D. (éd.), Modèles syntaxiques, Berne, Peter Lang, p. 279-296.

BlanCHE-BenVENISTE, Cl. (1983), «Examen de la notion de subordination », Recherches sur le français parlé, no4, p. 71-115.

BlanCHE-BenVENISTE, Cl. (1990), Le français parlé : études grammaticales, Paris, Editions du CNRS.

Blanche-Benveniste, Cl. (2000), Approches de la langue parlée en français, Paris, Ophrys.

Blanche-Benveniste, Cl. (2001), «Auxiliaires et degrés de 'verbalité'», Syntaxe et Sémantique, n०3, p. 75-97.

BlanCHE-Benveniste, Cl. (2002), «Macro-syntaxe et micro-syntaxe: les dispositifs de la rection verbale» in Andersen, H. L. \& H. Nølke (eds.) Macro-syntaxe et macro-sémantique. Actes du colloque international d'Arhus, 17-19 mai 2001, Bern, Peter Lang, coll. Sciences pour la communication, p. 95-118.

Blanche-Benveniste, Cl. (2010), Le français. Usages de la langue parlée (Avec la collaboration de Philippe Martin pour l'étude de la prosodie), Leuven-Paris, Peeters.

BRAUlT-SCAPPINI, S.-A. (2007), «Etude du dispositif d'extraction en 'c'est...qu', différenciation entre une relative en 'c'est...qu' et une proposition clivée », L'information grammaticale, n¹14, p. 53-56. (Présentation de thèse)

CAPPEAU, P. (2005), «Les indéfinis à l'aune de l'oral», SCOLIA, n²0, p. 67-82.

CAppeau, P. \& J. Deulofeu (2001), « Partition et topicalisation: il y en a 'stabilisateur' de sujets et de topiques indéfinis ", Cahiers de praxématique, n³7, p. 45-82.

Cappeau, P. \& J. Deulofeu (2006), «Le fonctionnement de divers quantifieurs indéfinis à l'oral » in Kleiber, G. \& al. (dir.), La relation partie-tout, Louvain-Paris, Peeters.

CONTI, V. (en cours), Les séquences en J'AI $X$ QUI $V$ : descriptions syntaxiques en contexte (thèse de doctorat effectuée sous la direction de Marie-José Béguelin à l’Université de Neuchâtel, Suisse). 
Conti, V. (2004), «A propos des configurations en $j^{\prime}$ ai $X$ qui...», Travaux neuchatelois de linguistique, n40, p. 151-163.

FURUKAWA, N. (1987), «Sylvie a les yeux bleus: construction à double thème», Linguisticae Investigationes, noXI-2, p. 283-302.

FurUKaWA, N. (1996), Grammaire de la prédication seconde, Louvain-la-Neuve, Duculot.

FURUKAWA, N. (2002), «Construction grammaticale et sous-détermination structurelle : autour de l'emploi attributif du verbe avoir », Le français moderne, no70-2, p. 129-144.

Furukawa, N. (2005), Pour une sémantique des constructions grammaticales. Thème et thématicité, Bruxelles, De Boeck - Duculot.

LAGAE, V. \& C. RougeT, 1998, "Quelques réflexions sur les relatives prédicatives » in Bilger, M., K. van den Eynde \& F. Gadet (dir.), Analyse linguistique et approches de l'oral. Recueil d'études offert en hommage à Claire Blanche-Benveniste, Louvain-Paris, Peeters, p. 313-325.

LAMBRECHT, K. (1994), Information structure and sentence form, Cambridge, Cambridge University Press, 1995.

LAmbrecht, K. (2000), "Prédication seconde et structure informationnelle: la relative de perception comme construction présentative », Langue française, nº127, p. 49-66.

LAMBrECHT, K. (2002), «Topic, focus and secondary predication. The french presentational relative construction » in Beyssade, C., R. Bok-Bennema, F. Drijkoningen \& P. Monachesi (dir.), Romance Languages and Linguistic Theory 2000, John Benjamins, p. 171-212.

LAMBRECHT, K. (2004), «Un système pour l'analyse de la structure informationnelle des phrases. L'exemple des constructions clivées» in Fernandez-Vest, J. \& S. CarterThomas (dir.), Structure informationnelle et particules énonciatives. Essai de typologie, Paris, L'Harmattan.

LÉARD, J.-M. (1992), Les gallicismes. Etude syntaxique et sémantique. Louvain-la-Neuve, Duculot.

Riegel, M., J.-CHR. PELLAT \& R. Rioul (2009[1994]), Grammaire méthodique du français, Paris, PUF.

RothenberG, M. (1979), «Les propositions relatives prédicatives et attributives : problème de linguistique française », Bulletin de la Société de linguistique de Paris, no74, p. 351-395.

Rouget, CHr. \& L. SALze (1986), «C'est ... qui, c'est ... que : le jeu des quatre familles », RSFP, no7, p. 117-139.

SANDFELD, Kr. (1936), Syntaxe du français contemporain. II : Les propositions subordonnées, Paris, Droz.

TELliER, Chr. (1992), «Remarques sur avoir attributif et possessif », Revue québécoise de linguistique, n²2, 1, p. 165-181.

Willems, D. \& M. Meulleman (2010), "Tl y a des gens ils viennent acheter des aspirines pour faire de l'ean gazense'. Sur les raisons d'être des structures parataxiques en il y a», in Béguelin, M.-J., M. Avanzi \& G. Corminboeuf (dir.), 2010, La Parataxe. Structures, marquages et exploitations discursives, Tome 2, Berne, Peter Lang, coll. Sciences pour la communication, p. 167-184.

\section{Corpus}

DELIC (2004), « Présentation du Corpus de Référence du Français Parlé», RSFP, n॰18, p. 11-42. 